3

4 5

6

7

8

9

10

\section{Consequences of resource competition for sex allocation and discriminative behaviors in}

\section{a hyperparasitoid wasp}

\author{
Bertanne Visser ${ }^{\mathrm{a}, b^{*}}$, Cécile Le Lann ${ }^{\mathrm{c}}$, Helen Snaas ${ }^{\mathrm{a}}$, Ian C.W. Hardy ${ }^{\mathrm{d}} \&$ Jeffrey A. Harvey ${ }^{\mathrm{a}}$
}

6

7

${ }^{a}$ Department of Terrestrial Ecology, Netherlands Institute of Ecology, Droevendaalsesteeg 10, 6708 PB,

Wageningen, the Netherlands

${ }^{\mathrm{b}}$ Current address: Institut de Recherche sur la Biologie de l'Insecte, Université de Tours, IRBI UMR CNRS

7261, Avenue Monge, 37200 Tours, France

${ }^{\mathrm{c}}$ Department of Ecological Science, Section Animal Ecology, VU University, De Boelelaan 1085, 1081 HV

Amsterdam, the Netherlands

${ }^{\mathrm{d}}$ School of Biosciences, University of Nottingham, Sutton Bonington Campus, Loughborough, Leicestershire LE12 5RD, United Kingdom

*Corresponding author: Institut de Recherche sur la Biologie de l'Insecte, Université de Tours, IRBI UMR CNRS 7261, Avenue Monge, 37200 Tours, France, tel. +33 24736 73 47, fax +33 2473669 66, email: bertannevisser@gmail.com

Accepted 26 ${ }^{\text {th }}$ August 2013

Visser B, Le Lann C, Snaas H, Hardy ICW \& Harvey JA 2014 Consequences of resource competition for sex allocation and discriminative behaviors in a hyperparasitoid wasp. Behavioral Ecology and Sociobiology 68:105-113 
Abstract

Population-wide mating patterns can select for equal parental investment in both sexes, but limiting resources, such as mates or developmental substrates, can increase competition leading to biased sex ratios in favor of either sex. Such competition for resources typically occurs in spatially structured populations, where dispersal is limited. Here, we investigate if and how resource competition affects sex allocation, discriminative behaviors and competitive interactions of the wingless hyperparasitoid Gelis acororum, which exploits patchily distributed hosts. We show that G. acororum sex ratios are male biased and that this is not a consequence of constrained reproduction by virgin females. Our results suggest that this pattern of reproductive investment, which is only rarely observed in parasitoids, is a consequence of resource limitation, in terms of hosts rather than mates. Further, G. acororum appears not to respond to intrinsic host quality or to prior oviposition in its host. When competing interspecifically for host resources, G. acororum outcompetes its congener G. agilis, but does so mainly when ovipositing on the host first. Overall, our results suggest that host resource limitation could be an important environmental factor shaping sex allocation in G. acororum, with competition taking place both intra- and interspecifically.

\section{Keywords}


It has been an enduring effort in evolutionary ecology to reveal how organisms' reproductive investment is shaped by their environment. Fisher (1930) was one of the first to explain sex ratio patterns, where equal investment in both sexes is maintained when allocation of resources into either sex conveys similar costs and benefits. Due to frequency dependence, the least common sex is favored by natural selection, ultimately leading to equal sex ratios in subsequent generations. A critical assumption in Fisher's sex ratio theory is population homogeneity, where mate competition is population-wide. In many circumstances, however, populations are structured in terms of mating opportunities. A classic example, local mate competition (LMC), was described by Hamilton (1967) where offspring of only a small number of mothers are confined to the same patch prior to mating and dispersal: malemale competition for mates selects for female biased progeny sex ratios. Alternatively, population heterogeneity can lead to competition for breeding substrates. Clark (1978) was the first to formally describe skewed sex ratios in favor of males in response to severe competition between related females for breeding areas in prosimian primates. Similar findings have been obtained also for other animals, such as marsupials, ungulates, birds and insects (West 2009). Contrary to LMC, it is the female that experiences severe competition for resources; hence local resource competition (LRC) for breeding substrates favors a proportionally higher investment in males over females (Strohm and Linsenmair 1997; Foitzik et al. 2010).

Patchy environments often increase the probability of encountering kin and interactions between related individuals underlie many theories of sex ratio evolution (West 2009). Restriction to certain patches does not solely involve groups of related individuals and patch exploitation by other, unrelated, individuals is likely quite common when many individuals exploit similar resources (Herre 1985). Indeed, in the context of LRC, Silk (1983) described how male-biased sex ratios were maintained in primates, even when larger group sizes were considered and where individuals faced competition from both related and unrelated conspecifics. Clearly, larger group sizes do not always lead to selection for unbiased sex ratios simply by providing more mating opportunities. On the contrary, competition may become even more intense (Silk 1983). Males or females may not only have to compete with direct relatives, but also with individuals of other species within their community. Alongside intra-specific competition, inter-specific interactions might further increase competitive pressures for access to similar and limiting resources, potentially distorting sex ratios. 
Competition for resources can shape patterns of reproductive allocation, but it can further affect the extent to which discriminative behaviors are needed to assess the quality or quantity of resources (West et al. 2002). Discriminative behaviors can be highly beneficial. For instance, the negative effects of inbreeding can be avoided when locally mating females can identify kin to avoid sib-mating (Ode et al. 1995; Lihoreau et al. 2007). Furthermore, the ability of a female to estimate the availability or quality of an oviposition substrate is critical for the survival of her offspring and therefore the mother's reproductive success (Godfray 1994). Such benefits are counteracted, however, by substantial energetic costs associated with increased discriminative abilities (DeWitt et al. 1998; Chevin et al. 2010; Auld et al. 2010). In Drosophila melanogaster, lines selected for increased recognition of high versus low quality oviposition substrates showed a clear decrease in egg laying behavior compared to controls; hence discrimination ability can negatively affect key fitness-related traits, such as fecundity (Mery and Kawecki 2002, 2004). Thus when competition is severe, the costs associated with discriminative abilities might outweigh their benefits, favoring indiscriminate behaviors (West et al. 2002).

Parasitic wasps are insects whose larvae develop in, or on the bodies of other arthropods (Godfray 1994) and are common model organisms in studying the evolution of sex ratios (Hamilton 1967; Trivers and Willard 1973; Charnov et al. 1981; Werren 1983; Godfray 1994; Somjee et al. 2010; Nelson and Greeff 2011; King and Kuban 2012). This is in part because their haplodiploid sex determination offers female parasitoids a choice of investing resources in males (haploid eggs) or females (diploid eggs) (Cook 1993). Discriminative behaviors are also welldocumented in hymenopteran parasitoids and nearly all tested species show some ability to discriminate between resources of different qualities, such as mates (Grant et al. 1980; Godfray and Cook 1997; Raychoudhury et al. 2010) and hosts (Vinson 1976; Godfray 1994; Vinson et al. 1998; van Baaren et al. 2009). Furthermore, many parasitoid species compete heavily for resources, both within and between species (Price 1972; Harvey et al. 2013). For example, different developmental stages of the host, such as eggs or larvae, often harbor their own parasitoid guilds (Price 1972) and both within and between these guilds competition for host resources can be intense, particularly for solitary parasitoids where only one individual can emerge from a single host (Godfray 1994).

Here, we study the solitary, wingless hyperparasitoid Gelis acororum (Hymenoptera: Ichneumonidae) that commonly attacks patchily distributed gregarious hosts on the ground and in low vegetation. Winglessness reduces the dispersal potential and the patchy distribution of clusters of hosts leads this parasitoid exploit groups of separate hosts together, in a manner termed 'quasi-gregarious' (e.g. Hardy 1992, Godfray 1994). Female-biased sex ratios 
are prevalent amongst gregarious and quasi-gregarious parasitoids and may result from inbreeding or competition between males for access to mates (LMC), (Godfray 1994, West 2009). To establish whether females avoid inbreeding, we first performed mate choice experiments using related or unrelated males. We then tested for LMC by determining sex allocation patterns, following mating with a related or an unrelated male. In contrast to the female bias often observed due to LMC, sex ratios were male biased. The remainder of our experiments were designed to test candidate explanations for male-biased sex ratios. We first confirmed that similar sex ratio patterns were observed among the progeny of a larger number of females whose mating was not experimentally controlled. We then assessed whether G. acororum is able to recognize and discriminate against hosts of different quality and prior parasitism status, as reduced discriminative behaviours are expected under strong competitive pressure. Finally, we determined G. acororum's competitive ability when competing for resources with its congener Gelis agilis, this allowed us to estimate the competitive pressure posed by a species with which it naturally co-occurs and which utilizes similar host resources.

\section{Materials and methods}

\section{Origin and rearing of insects}

The primary endoparasitoid Cotesia glomerata (Hymenoptera: Braconidae) and its host Pieris brassicae (Lepidoptera: Pieridae) were obtained from existing cultures at the University of Wageningen (WUR, the Netherlands) and were originally collected from agricultural fields near the university. Cotesia glomerata cultures were maintained as described in Harvey (2000). Gelis acororum and G.agilis were collected from C. glomerata cocoon clutches placed on a garden plot adjacent to the Netherlands Institute of Ecology in Wageningen, the Netherlands. Cultures were maintained within population cages at a constant temperature of $25 \pm 1{ }^{\circ} \mathrm{C}$, a $16: 8 \mathrm{~L}: \mathrm{D}$ regime and a relative humidity of $65 \% \pm 10$ with access to honey and water on cotton wool. Both species were maintained on 1-2 day-old C. glomerata pupae and allowed to host-feed. Host-feeding is essential for egg maturation in G. agilis (Harvey 2007; Harvey et al. 2011) and has also been observed in G. acororum (Pers. obs. B. Visser, J. Harvey). Similar to G. agilis, host-feeding likely enhances the rate of egg maturation in G. acororum and could be essential for maintaining egg maturation later in life; hence all G. acororum females were allowed to host-feed. For experiments, abiotic conditions were similar to that of the cultures, but all behavioral observations were performed at a temperature of $20 \pm 1^{\circ} \mathrm{C}$. 
To obtain individuals of known family status, females of a range of ages were obtained from population cages and placed singly in small Petri dishes $(\varnothing=6 \mathrm{~cm})$. Each female was allowed access to a clutch containing approximately 25-35 C. glomerata cocoons, honey and water on cotton wool for 4 days (approximating the period during which the cocoons are suitable for parasitism). After 1 week of development, unparasitized cocoons from which $C$. glomerata had emerged were removed, and cocoons containing G. acororum progeny were placed singly in small Petri dishes. Within one week after adult emergence, mate preference for a brother or an unrelated male was tested for 20 host-fed G. acororum females. For these mate-choice experiments, a brother and an unrelated male were introduced into a small Petri dish first, followed by introduction of the female. All interactions were videotaped for 24 minutes or until mating was observed, after which mate-choice was determined by tracking individuals.

\section{Sex allocation patterns through local mate or resource competition}

To test if females adjusted sex ratios following sib-mating, additional pairs were allowed to mate. A total of 13 females that had mated with a brother and 9 females that had mated with an unrelated male were transferred to a large Petri dish $(\varnothing=12 \mathrm{~cm})$ and offered $351-2$ day old $C$. glomerata cocoons, as well as honey and water on cotton wool for a duration of 4 days. Secondary sex ratios were determined two weeks later, following adult eclosion.

Sex ratios were further determined for the progeny of 81 host-fed females from population cages, where each female had access to approximately 25-35 C. glomerata cocoons, honey and water on cotton wool during 4 days. Cocoon clutches far exceeded the number of cocoons that can maximally be parasitized by G. acororum and contained an average of 28 (S.E. \pm 0.33 ) C. glomerata cocoons. On average 15 (S.E. \pm 0.72 ) of these cocoons remained unparasitized by G. acororum, leading to emergence of the host $C$. glomerata. An average of 4 (S.E. \pm 0.30 ) G. acororum offspring developed successfully.

\section{Discriminative abilities}

\section{External host marking and internal host discrimination}

In several parasitoid species, females are known to leave a chemical mark on their host after parasitism, lasting up to several hours, and which is typically used to signal the presence of an egg to a conspecific female (Nufio and Papaj 2001). To establish whether G. acororum discriminated between cocoons previously parasitized by itself or 
glomerata cocoon. Following oviposition the cocoon was immediately removed and placed into a small Petri dish together with a cocoon that had been parasitized by an unrelated female. Each female was then introduced into the Petri dish and allowed to choose during 1 hour or until oviposition occurred between a cocoon parasitized by itself or a cocoon parasitized by an unrelated female.

170

Unlike species that can detect external marking, some parasitoids can deduce parasitism status of the host only after probing it with the ovipositor, for instance through detecting physical or biochemical changes on the host surface or in the host body itself (King and Rafai 1970). In subsequent experiments, females were offered hosts containing G. acororum progeny that had developed until the pre-pupal stage. To obtain cocoons parasitized by G. acororum, females from population cages were placed singly in a small Petri dish with access to 1 C. glomerata cocoon. Each female was observed for 6 hours or until oviposition occurred. Progeny were then allowed to develop until the pre-pupal stage over the following 7 days. To test whether or not females could discriminate parasitized from unparasitized cocoons, a total of 21 females were allowed to choose between a cocoon containing a $G$. acororum pre-pupa and a 1-2 day old unparasitized cocoon containing the host C. glomerata.

Viability assessment

To determine if females were able to assess viability of the host (i.e. whether it was alive or dead), 1-2 day-old $C$. glomerata cocoons were frozen at $-18^{\circ} \mathrm{C}$ for 3 days. Prior to the experiments cocoons were left at room temperature for at least one hour. 19 females were then placed singly in a small Petri dish and offered a choice between a live and dead host for 6 hours or until oviposition occurred.

Intra-specific host recognition

To evaluate intra-specific discriminative ability of G. acororum, female preference for cocoons containing 7 dayold progeny varying in the level of relatedness was determined using three two-choice combinations: 1) A cocoon containing the female's own progeny vs. a cocoon containing her sister's progeny $(\mathrm{n}=14) ; 2)$ the female's own progeny vs. progeny of an unrelated female $(n=18) ; 3$ ) her sister's progeny vs. progeny of an unrelated female $(n=16)$. For each experiment, a single female was allowed to choose for 6 hours or until oviposition occurred. 
To determine larval survival when $G$. acororum is competing with its sister species G. agilis, single females of both species were allowed to oviposit on 1 C. glomerata cocoon for 6 hours or until oviposition occurred. Cocoons parasitized by G. acororum were then offered to G. agilis $1(\mathrm{n}=46), 3(\mathrm{n}=31)$ and 7 days $(\mathrm{n}=22)$ after initial oviposition and observed for 6 hours or until oviposition occurred. To evaluate competitive ability of $\boldsymbol{G}$. acororum when offspring had a developmental disadvantage (i.e. the competitor is at a more advanced developmental stage), reciprocal experiments were also performed, in which G. agilis oviposited first, followed by $G$. acororum ( $\mathrm{n}=24,12$ and 18 after 1,3 and 7 days, respectively). After two weeks of development, emergence was monitored and species identity recorded.

\section{Statistics}

Female preferences for an unrelated or related male were determined using a Binomial test. Sex allocation patterns were analyzed using generalized linear models assuming quasi-binomially distributed error variances (logistic analyses for quasi-brood sex ratios) and quasi-Poisson distributed error variances (log-linear analyses for numbers of males per quasi-brood) with significance assessed through model simplification (Crawley 1993; Wilson and Hardy 2002). Meelis tests were used to assess sex ratio variances: the significance of any deviation from binomiality for sex ratio and mortality data was assessed using the test statistic, $U$. The associated variance ratio, $R$, was used as a quantification of variance, where overdispersion is indicated by $R>1$ and underdispersion by $R$ < 1 (Nagelkerke and Sabelis 1991; Krackow et al. 2002). Data on host choice preferences and differential survival in competition experiments were analyzed using Binomial tests. GLM's were performed using the statistical software package GenStat version 15 (VSN International) and binomial tests were performed using R software version 2.12.1 (R Development Core Team 2010).

\section{Results}

\section{Inbreeding avoidance and sex allocation patterns through local mate or resource competition}

In mate-choice experiments, females did not show a preference for either a brother or an unrelated male. Out of 20 females for which mate-choice was assessed, half chose a brother as a mate, whereas the other half chose an unrelated male (Binomial test: $\mathrm{p}=1)$. We found no evidence of sex ratio adjustment following sib-mating $\left(F_{1,20}=\right.$ $1.69, p=0.210)$, no relationship between sex ratio and quasi-brood size $\left(F_{1,19}=0.15, p=0.703\right.$, Fig. $\left.1 \mathrm{a}\right)$ nor an interaction between these main effects $\left(F_{1,18}=0.16, p=0.697\right)$. The number of males per quasi-brood increased significantly with increasing quasi-brood size $\left(F_{1,20}=33.74, p<0.001\right.$, Fig. 1c). Quasi-brood sex ratios were 
overdispersed (quasi-broods $>1, \mathrm{n}=19, U=4.43, \mathrm{p}<0.001, R=3.924$ ) and strongly male-biased (mean proportion of males: $0.810,+\mathrm{SE}=0.053,-\mathrm{SE}=0.068$ ). Our results further showed that $55 \%$ of all females produced single-sex male quasi-broods, $9 \%$ produced female-only quasi-broods and 36\% produced mixed-sex quasi-broods.

A total of 331 individuals emerged out of 81 quasi-broods of females from population cages: 221 males and 110 females. Sex ratios were male-biased (mean proportion of males: $0.668,+\mathrm{SE}=0.044,-\mathrm{SE}=0.047$ ). Sex ratio variance was overdispersed when all quasi-broods $>1$ were considered $(\mathrm{n}=69, U=8.61, p<0.001, R=2.775$ ) but did not differ significantly from binomiality when data from only mixed sex quasi-broods were considered (n $=25, U=-0.89, \mathrm{p}>0.05, R=0.865)$. For all 81 quasi-broods, there was no relationship between sex ratio and quasi-brood size $\left(F_{1,79,}=0.01, \mathrm{p}=0.926\right.$, Fig. $\left.1 \mathrm{~b}\right)$ and a similar result was found for mixed sex quasi-broods only $\left(F_{1,24},=0.19, p=0.668\right)$. For both sets of data, the number of males increased significantly with increasing quasibrood size (all quasi-broods: $F_{1,79},=68.46, \mathrm{p}<0.001$, Fig. $1 \mathrm{~d}$; mixed sex quasi-broods: $F_{1,24}=18.71, \mathrm{p}<0.001$ ). Among all quasi-broods, $51 \%$ of females produced single-sex male quasi-broods only, $17 \%$ produced female-only quasi-broods and $32 \%$ produced mixed-sex quasi-broods.

\section{Discriminative abilities}

\section{External host marking and internal host discrimination}

When females were offered a choice between cocoons recently parasitized by themselves or an unrelated female, 13 out of 22 females chose their own cocoon over an unrelated cocoon (Binomial test: $\mathrm{p}=0.524$ ), suggesting that G. acororum does not mark its host to avoid self-parasitism. Females also did not prefer unparasitized over parasitized cocoons: 10 females oviposited in unparasitized cocoons, while 11 females oviposited in parasitized cocoons (Binomial test: $\mathrm{p}=1$; Fig. 1a).

\section{Viability assessment}

Choice tests evaluating the ability of G. acororum to assess host viability showed that 10 out of 19 females oviposited on dead C. glomerata cocoons (Binomial test: $\mathrm{p}=1$; Fig. 1a); hence G. acororum females do not discriminate against, or avoid ovipositing on, dead hosts.

\section{Intra-specific host recognition}


Females did not discriminate between cocoons containing progeny that varied in the degree of relatedness. Eight out of 14 females chose their own cocoon over that of their sister's (Binomial test: $p=0.791), 7$ out of 18 preferred their own cocoon over that of an unrelated female (Binomial test: $p=0.481$ ) and 8 out of 16 females chose their sister's cocoon when offered a choice between their sister's cocoon and that of an unrelated female (Binomial test: $\mathrm{p}=1$; Fig. 1b).

\section{Competitive abilities}

Survival of G. acororum was higher than that of G. agilis when G. acororum was the first to parasitize a common host cocoon: The percentage of G. acororum individuals surviving to adulthood increased from 74 to 77 and $91 \%$ as time elapsed between oviposition events increased (1, 3 and 7 days, respectively: Binomial tests: 1 day $p<$ $0.01, \mathrm{df}=46 ; 3$ days $\mathrm{p}<0.01, \mathrm{df}=31 ; 7$ days $\mathrm{p}<0.001, \mathrm{df}=22 ;$ Fig. 2a). In contrast, when G. agilis oviposited first, we found either no difference in survival (after 1 day: $\mathrm{p}=0.839$, df $=24$ ), a competitive advantage for $G$. agilis (after 3 days: $\mathrm{p}=0.039, \mathrm{df}=12$ ) or higher survival into adulthood for $G$. acororum (after 7 days: $\mathrm{p}<0.001$, df $=18 ;$ Fig. 2b)

\section{Discussion}

Mate-choice experiments did not reveal a preference of females in terms of mating with their brother or an unrelated male. Avoidance of sib-mating can reduce the negative effects associated with inbreeding, such as increased haploid male unviability (Heimpel and de Boer 2008); hence inbreeding avoidance could be beneficial. We did, however, not find evidence that G. acororum females avoid inbreeding through mate-choice. Females further did not adjust sex ratios in favor of female offspring once they had mated with a brother. Sex ratio adjustment in favor of females could be expected if males that disperse post-mating are faced with intense competition over access to females (Hamilton 1967; Macke et al. 2011). We thus found no evidence for LMC in G. acororum and the absence of inbreeding avoidance and LMC suggests that genetic effects of inbreeding might be minimal in this species or that $G$. acororum outbreeds sufficiently to avoid the negative consequences of inbreeding.

G. acororum sex ratios are biased in favor of males; yet most theoretical models on optimal sex allocation patterns, particularly in parasitoids, predict female-biased sex ratios (Hamilton 1967; Charnov et al. 1981; Werren 1983; West 2009). Several conditions may, however, promote a higher investment in males, such as host limitation 
through dense rearing conditions (Heimpel and Lundgren 2000; Ode and Heinz 2002) and a high number of foundresses (Hamilton 1967; Werren 1983; Debout et al. 2002). It seems unlikely that these factors contributed to the male-biased sex ratios we observed, first because G. acororum had only been reared in the laboratory for a limited number of generations and population cages were provided with a surplus of hosts. Second, females were allowed to oviposit on a host cocoon singly during experiments; hence foundress number was fixed at one. Furthermore, under high host or parasitoid density, even if males can become more numerous within the population (i.e. sex ratios increase), the overall sex ratio typically remains female-biased, in contrast to our observations on G. acororum where the majority of individuals are males.

Haplodiploidy in parasitoids allows females to manipulate investment in each sex through the decision of whether or not to fertilize an egg; hence females can adjust the sex ratio of their offspring at the time of oviposition. An important factor affecting sex ratio decisions in parasitoids is host size or quality; typically, mothers preferentially invest in female offspring when hosts are larger (Charnov et al. 1981; Tanaka et al. 1992; Ueno 1997; Ode and Heinz 2002). Male-biased sex ratios are, however, maintained when G. acororum oviposits on a larger host species, Cotesia rubecula, even though G. acororum preferred this host over C. glomerata (Pers. Obs. B. Visser, J. Harvey). Alternatively, males can become more abundant through differential mortality of the sexes, even when females invest equally in both sexes during oviposition (Hardy 1992; King 1993; Hardy et al. 1998). Mechanisms that promote sex-specific mortality include inter-sexual conflict (Kapranas et al., 2011) and the potential for increased exposure of detrimental mutations in haploids (Nagelkerke and Hardy 1994). In sexually size dimorphic parasitoids, females can experience higher mortality in smaller or lower quality hosts, leading to a higher number of males (Charnov et al. 1981; Godfray 1994). Size does, however, not differ between the sexes in G. acororum (Pers. obs. B. Visser, J. Harvey) making it unlikely that survival of the sexes is differentially affected by host size in this species.

Male-biased sex ratios in G. acororum suggest that LRC might play an important role in this species, where females compete heavily for access to host resources. Over $50 \%$ of all quasi-broods produced by G. acororum are single-sex and male-only. Single-sex male and female quasi-broods have also been observed in several parasitoid species within the genus Achrysocharoides (West et al. 1999). For example, A. zwoelferi produces single-sex clutches only (West et al. 2001). The oviposition strategy of this wasp is exceptional, however, because brood sizes are small and male-only broods have an invariable clutch size of 1 . Single-sex nests were also observed in 
the sphecid Trypoxylon malaisei, although these constituted only 5\% of nests observed (Oku and Nishida 2001).

The most straightforward explanation for the occurrence of male-only broods in $G$. acororum would be that females remained unmated (Hardy et al. 1998). However, mating was observed during experiments testing for sex ratio adjustment through LMC; hence lack of mating does not explain the common production of male-only $G$. acororum broods. We have yet to identify which conditions affect the decision-making process of females in this species and what the adaptive significance is of producing male-only broods.

Skewed sex ratios in favor of males can also result when mothers are constrained in sperm usage, for instance when the efficiency of sperm transfer or storage is reduced. In such scenarios, mated females may not be able to fertilize sufficient eggs, leading to a predominance of males. In some parasitoids a single mating suffices to assure continued production of females, despite a reduction in sperm numbers stored over time (Bressac and Chevrier 1998), whilst others require multiple matings to avoid sperm depletion (Ode et al., 1997). Our comparison of sex ratios between recently emerged females and population-cage females of a range of ages showed similar sex ratio patterns (Fig. 1), suggesting that sex ratios and the number of male-only quasi-broods produced is are not dependent on female age. Whilst population-cage females are capable of re-mating, female G. acororum with limited mating opportunities early during adult life have been observed to produce daughters at more advanced ages (Pers. Obs. B. Visser, J. Harvey), suggesting that sperm depletion plays little or no role in the sexual composition of quasi-broods.

Individuals can avoid LRC, for instance through dispersal (Silk and Brown 2008; Guillon and Bottein 2011). A clear example can be found in the parasitoid Mellitobia australica (Innocent et al. 2010), a species that has two distinct female morphs: one that is better equipped for dispersal (longer wings), and the other better suited for remaining in the natal patch (shorter wings). Indeed, when intra-specific competition for resources increases, $M$. australica females invest more heavily in long-winged dispersing morphs. However, when individuals compete with relatives, conspecifics (Clark 1978; Silk 1983; Silk 1984; Silk and Brown 2008) as well as other species, dispersal is unlikely to relieve competitive pressures when community composition remains relatively stable. Winglessness in G. acororum reduces its dispersal potential in the field, and unlike males that compete solely with conspecifics for access to mates, females are faced with competition for resources with other parasitoid species that share the same hosts (Harvey et al. 2011; 2013). Within stable dense communities, competition can thus be 
hard to avoid, and multi-species interactions can further contribute to the distorted sex ratios observed in $G$. acororum.

Females did not show an egg-laying preference for parasitized or unparasitized hosts, hosts that were alive or dead, or cocoons containing progeny varying in the level of relatedness. We thus found no evidence of host and intraspecific discrimination, or an ability to assess host viability. In hymenopteran parasitoids, a complete absence of discriminative behaviors has only rarely been described and such findings have often been questioned or refuted (Lenteren et al. 1978; van Alphen and Visser 1990). Absence of discriminative behaviors in G. acororum might suggest that certain host types are rarely encountered in nature, and that selection regimes have not been rigid enough to select for the avoidance of unsuitable hosts. Sait et al. (1996) found that the solitary endoparasitoid, Venturia canescens, was unable to discriminate between healthy host caterpillars (Plodia interpunctella) and caterpillars heavily infested with a granulosis virus, and readily oviposited into the latter. However, parasitoids were unable to survive to eclosion in hosts exhibiting latent viral infection. Gelis acororum does discriminate when offered a choice between a $C$. glomerata cocoon and a cocoon of the larger host $C$. rubecula, where the latter is favored for oviposition (Harvey et al. in prep). Gelis acororum thus seems capable of determining either host species identity or marked size differences, but ceases to actively discriminate against different host states when only $C$. glomerata cocoons are available.

Gelis acororum outcompeted its congener G. agilis when allowed to oviposit first. When G. agilis oviposited first, survival of both species was similar after 1 day, in favor of G. agilis after 3 days but reversed in favor of $G$. acororum after 7 days. Both species are wingless and share similar resources. G. acororum has a high abundance in the field (Harvey et al., in prep) and this is reflected by the success with which it outcompetes its main competitor G. agilis. While this study is the first to investigate competitive interactions between sibling species in the genus Gelis, a previous study on G. agilis showed that it outcompetes the more specialized solitary pupal hyperparasitoid, Lysibia nana (Hymenoptea: Ichneumonidae). After more than 72 hours after initial oviposition, G. agilis always dominates, leading to a trophic switch for this species in which it can successfully exploit another hyperparasitoid as a host later in the season (Harvey et al. 2011). The timing of oviposition is critical for survival and competitive success in G. acororum and in the field temporal variability in host availability and number of competitors are expected to pose major competitive challenges for both species of Gelis. 
The male-biased sex ratios observed in G. acororum are very unusual among parasitoids, and a combination of low discriminative behaviors and high competitive pressure indicate the possibility of extensive resource competition between females in the field. Such interactions likely occur between conspecifics, but within dense communities where many co-occurring species exploit similar resources, females may also face adverse conditions that are imposed by females of other species. These considerations are lacking in current theories concerned with sex ratio evolution, yet community composition, of both parasitoids and of hosts (Kraft \& van Nouhuys 2013) might substantially affect resource availability and inter-specific competition, consequently affecting key traits, such as reproductive success mediated by sex allocation decisions.

\section{Acknowledgements}

We are grateful to Flavia Araujo Barbosa and two anonymous referees for providing helpful comments on our manuscript and we would like to thank Roel Wagenaar for his rearing of the hosts and parasitoids.

\section{References}

Alphen JJM van, Visser ME (1990) Superparasitism as an adaptive strategy for insect parasitoids. Ann Rev Entomol 35:59-79

Auld JR, Agrawal AA, Relyea RA (2010) Re-evaluating the costs and limits of adaptive phenotypic plasticity. Proc Roy Soc B 277:503-11

Baaren J van, Le Lann C, Pinchenot J, Pierre JS, Krespi L, Outreman Y (2009) How could host discrimination abilities influence the structure of a parasitoid community? Bull Entomol Res 99:299-306

Charnov EL, Los-den Hartogh RL, Jones WT, Assem J van den (1981) Sex ratio evolution in a variable environment. Nature 289:27-33

Chevin L-M, Lande R, Mace GM (2010) Adaptation, plasticity, and extinction in a changing environment: Towards a predictive theory. PLoS Biol 8:e1000357

Clark AB (1978) Sex ratio and local resource competition in a prosimian primate. Science 201:163-165

Cook JM (1993) Sex determination in the Hymenoptera: A review of models and evidence. Heredity 71:421-435

Crawley MJ (1993) GLIM for Ecologists. Blackwell Scientific Publications. 
Debout G, Fauvergue X, Fleury F (2002) The effect of foundress number on sex ratio under partial local mate competition. Ecol Entomol 27:242-246

Fisher RA (1930) The genetical theory of natural selection. Oxford University Press.

Foitzik S, Kureck IM, Rüger MH, Metzler D (2010) Alternative reproductive tactics and the impact of local competition on sex ratios in the ant Hypoponera opacior. Behav Ecol Sociobiol 64:1641-1654

Godfray HCJ (1994) Parasitoids: behavioral and evolutionary ecology. Princeton University Press.

Godfray HCJ, Cook JM (1997) Mating systems of parasitoid wasps. In: Choe JC, Crespi BJ (eds) The evolution of mating systems in insects and arachnids. Cambridge University Press, pp 211-225.

Grant B, Burton S, Contoreggi C, Rothstein M (1980) Outbreeding via frequency-dependent mate selection in the parasitoid wasp, Nasonia (=Mormoniella) vitripennis Walker. Evolution 34:983-992

Guillon JM, Bottein J (2011) A spatially explicit model of sex ratio evolution in response to sex-biased dispersal. Theor Pop Biol 80:141-149

Hamilton WD (1967) Extraordinary sex ratios. Science 156:477-488

Hardy ICW (1992) Nonbionomial sex allocation and brood sex-ratio variances in the parasitoid Hymenoptera.

Oikos 65:143-158

Hardy ICW, Dijkstra LJ, Gillis JEM, Luft PA (1998) Patterns of sex ratio, virginity and developmental mortality in gregarious parasitoids. Biol J Linn Soc 64:239-270

Harvey JA (2000) Dynamic effects of parasitism by an endoparasitoid wasp on the development of two host species: Implications for host quality and parasitoid fitness. Ecol Entomol 25:267-278

Harvey JA (2007) Comparing and contrasting development and reproductive strategies in the pupal hyperparasitoids Lysibia nana and Gelis agilis (Hymenoptera: Ichneumonidae). Evol Ecol 22:153-166

Harvey JA, Poelman EH, Tanaka T (2013) Intrinsic inter- and intra-specific competition in parasitoid wasps. Ann Rev Entomol 58:333-351 

hyperaparasitoids results in temporal trophic switch. Oikos 120: 226-233.

Herre EA (1985) Sex ratio adjustment in fig wasps. Science 228:896-898

Innocent TM, Abe J, West SA, Reece SE (2010) Competition between relatives and the evolution of dispersal in a parasitoid wasp. J Evol Biol 23:1374-85

Kapranas A, Hardy ICW, Morse JG, Luck RF (2011) Parasitoid developmental mortality in the field: patterns, causes and consequences for sex ratio and virginity. J Anim Ecol 80:192-203 of sex ratio in insects and mites. Chapman \& Hall, New York, pp 418-441.

King BH, Kuban KA (2012) Should he stay or should he go: male influence on offspring sex ratio via postcopulatory attendance. Behav Ecol Sociobiol 66:1165-1173

King PE, Rafai J (1970) Host discrimination in a gregarious parasitoid Nasonia vitripennis (Walker) (Hymenoptera: Pteromalidae). J Exp Biol 53:245-254

Krackow S, Meelis E, Hardy ICW (2002) Analysis of sex ratio variances and sequences of sex allocation. In:

441 Hardy ICW (ed) Sex Ratios: Concepts and Research Methods. Cambridge University Press, pp 112-131.

442 Lenteren JC, Bakker K, Alphen JJM van (1978) How to analyse host discrimination. Ecol Entomol 3:71-75

443 Lihoreau M, Zimmer C, Rivault C (2007) Kin recognition and incest avoidance in a group-living insect. Behav $444 \quad$ Ecol 18:880-887

445 Macke E, Magalhães S, Bach F, Olivieri I (2011) Experimental evolution of reduced sex ratio adjustment under 446 local mate competition. Science 334:1127-1129

447 Mery F, Kawecki TJ (2002) Experimental evolution of learning ability in fruit flies. Proc Natl Acad Sci USA 
Nagelkerke CJ, Sabelis MW (1991) Precise sex ratio control in the pseudo-arrhenotokous phytoseiid mite Typhlodromus occidentalis Nesbitt. In: Schuster R, Marphy PW (eds) The Acari: Reproduction, development and life-history strategies. Chapman \& Hall, pp 193-207.

Nagelkerke CJ, Hardy ICW (1994) The influence of developmental mortality on optimal sex allocation under local mate competition. Behav Ecol 5:401-411

Nelson RM, Greeff JM (2011) Sex ratio dependent dispersal when sex ratios vary between patches. J Theor Biol

Nufio CR, Papaj DR (2001) Host marking behavior in phytophagous insects and parasitoids. Entomol Exp Appl 99:273-293

Ode PJ, Antolin MF, Strand MR (1995) Brood-mate avoidance in the parasitic wasp Bracon hebetor Say. Anim

Ode PJ, Heinz KM (2002) Host-size-dependent sex ratio theory and improving mass-reared parasitoid sex ratios.

Oku S, Nishida T (2001) Presence of single-sex broods under local mate competition in Trypoxylon malaisei (Hymenoptera: Sphecidae): Adaptation or maladaptation? Ann Entomol Soc Am 94:550-554

Price PW (1972) Parasitoids utilizing the same host: Adaptive nature of differences in size and form. Ecology $53: 77-78$

467 Raychoudhury R, Desjardins CA, Buellesbach J, Loehlin DW, Grillenberger BK, Beukeboom L, Schmitt T, 468 Werren JH (2010) Behavioral and genetic characteristics of a new species of Nasonia. Heredity 104:278-88

469 R Development Core Team (2010) R: A language and environment for statistical computing. R Foundation for 470 Statistical Computing, Vienna, Austria, ISBN 3-900051-07-0. Available from: http://www.R-project.org/.

471 Sait SM, Begon M, Thompson DJ, Harvey JA (1996) Parasitism of baculovirus-infected Plodia interpunctella by

472 Venturia canescens and subsequent virus transmission. Funct Ecol 10:586-591 
Silk JB (1983) Local resource competition and facultative adjustment of sex ratios in relation to competitive abilities. Am Nat 121:56-66

Silk JB (1984) Local resource competition and the evolution of male-biased sex ratios. J Theor Biol 108:203-13

Silk JB, Brown GR (2008) Local resource competition and local resource enhancement shape primate birth sex ratios. Proc Roy Soc B 275:1761-5

Somjee U, Ablard K, Crespi B, Schaefer PW, Gries G (2010) Local mate competition in the solitary parasitoid wasp Ooencyrtus kuvanae. Behav Ecol Sociobiol 65:1071-1077

Strohm E, Linsenmair KE (1997) Low resource availability causes extremely male-biased investment ratios in the 179:90-92

Ueno T (1997) Host age preference and sex allocation in the pupal parasitoid Itoplectis naranyae (Hymenoptera: Ichneumonidae). Ann Entomol Soc Am 90:640-645 sex broods. Anim Behav 57:265-275 strategy of a parasitoid wasp that produces single sex clutches. Behav Ecol 12:577-583 
497 Wilson K, Hardy ICW (2002) Statistical analysis of sex ratios: an introduction. In: Hardy ICW (ed) Sex Ratios: 498 Concepts and Research Methods. Cambridge University Press, pp 48-92.

499

500 

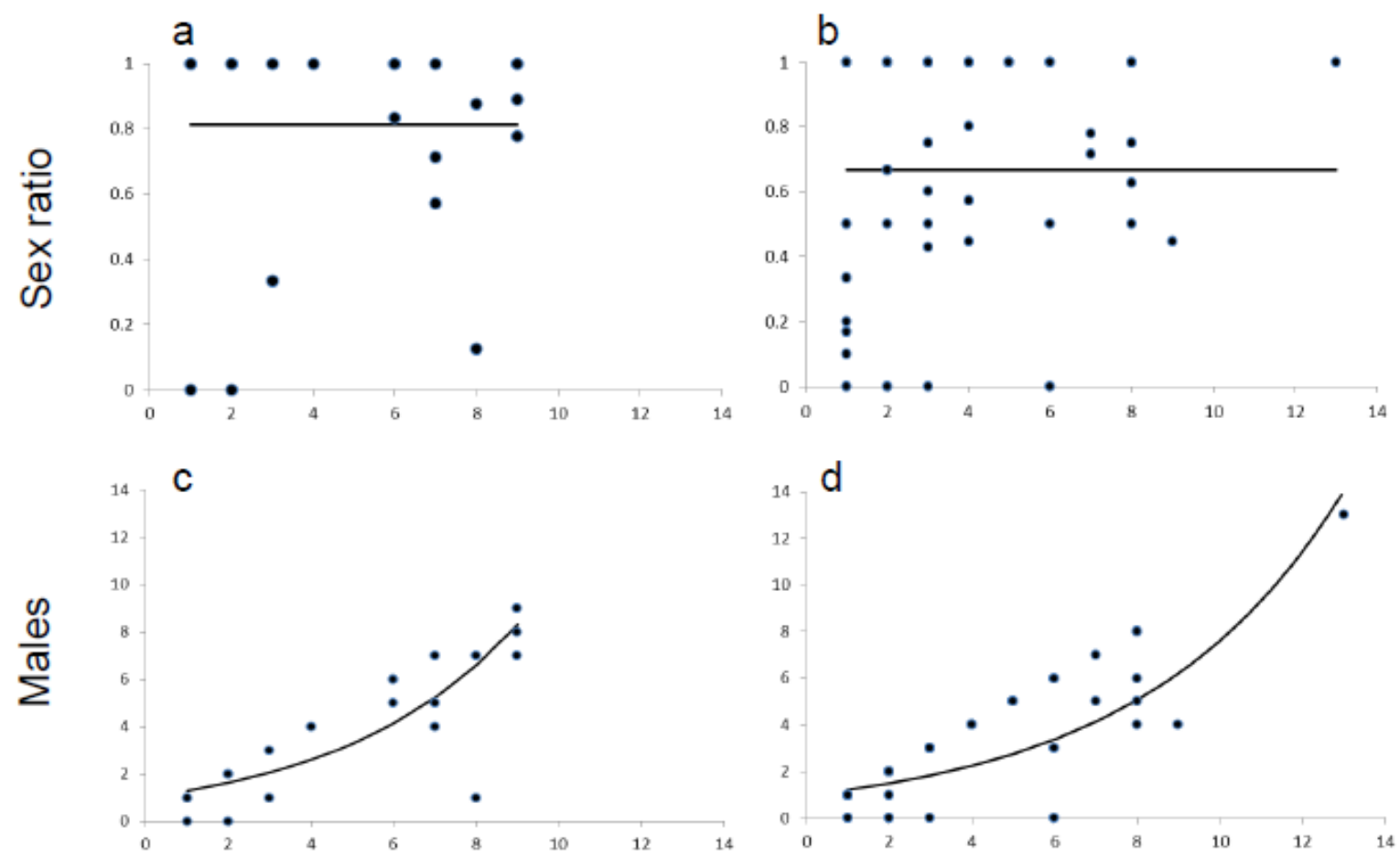

\section{Brood size}

503

504 Fig. 1: Sexual composition of Gelis acrorum quasi-broods. Sex ratios are shown from the mate choice experiment quasi-broods (a) and from quasi-broods from population cages (b). The relationship between the number of males and quasi-brood size is shown for the mate choice experiment (c) and for all population cage quasi-broods (d). On all panels regression lines represent the minimal adequate statistical model (logistic analyses, panels a and b; loglinear analyses, panels $\mathrm{c}$ and d). 

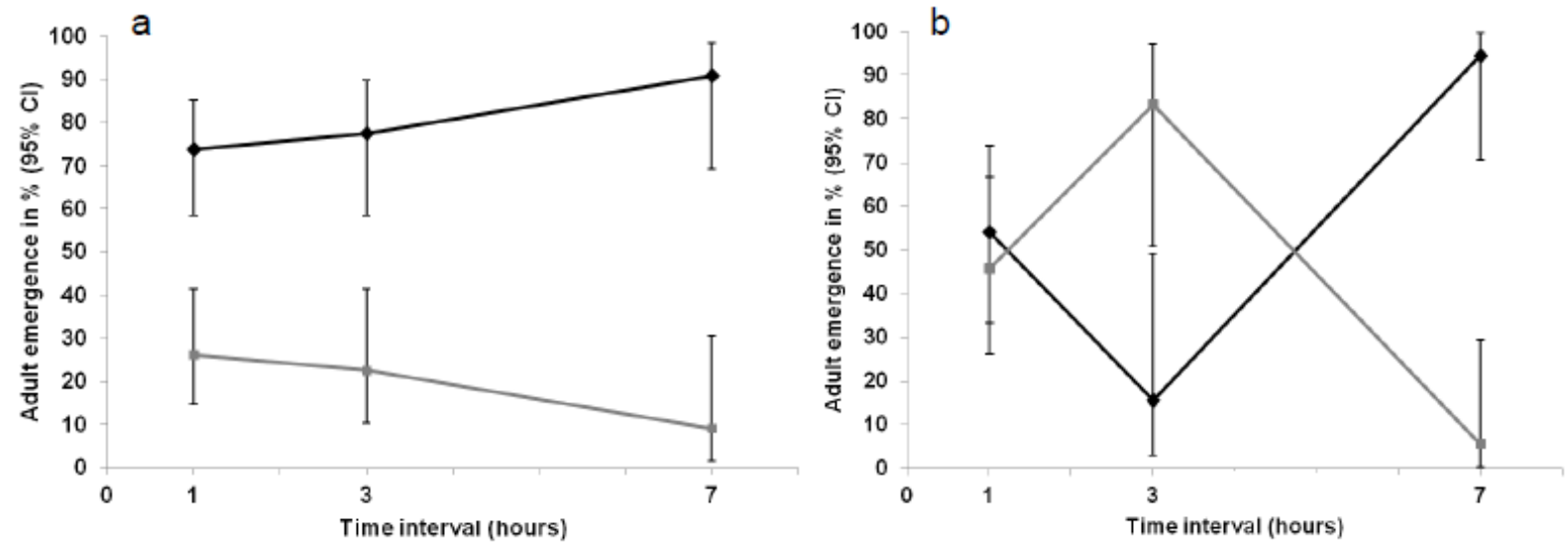

517 Fig. 2: Outcome of intrinsic competition (in percentage of adults that emerged including Wilson's 95\% Confidence

518 Interval with continuity correction) between G. acororum (black dots) and G. agilis (gray dots) 1, 3 and 7 days

519 after initial oviposition with either G. acororum parasitizing first (a) or G. agilis parasitizing first (b). 